\title{
Reflexiones sobre los retos de la política educativa en el Caribe colombiano: una indagación en prensa
}

\section{Some reflections about challenges of education policies in the Colombian Caribbean: a media analysis}

Juan David Parra* y Gustavo Correa Hernández ${ }^{* * *}$

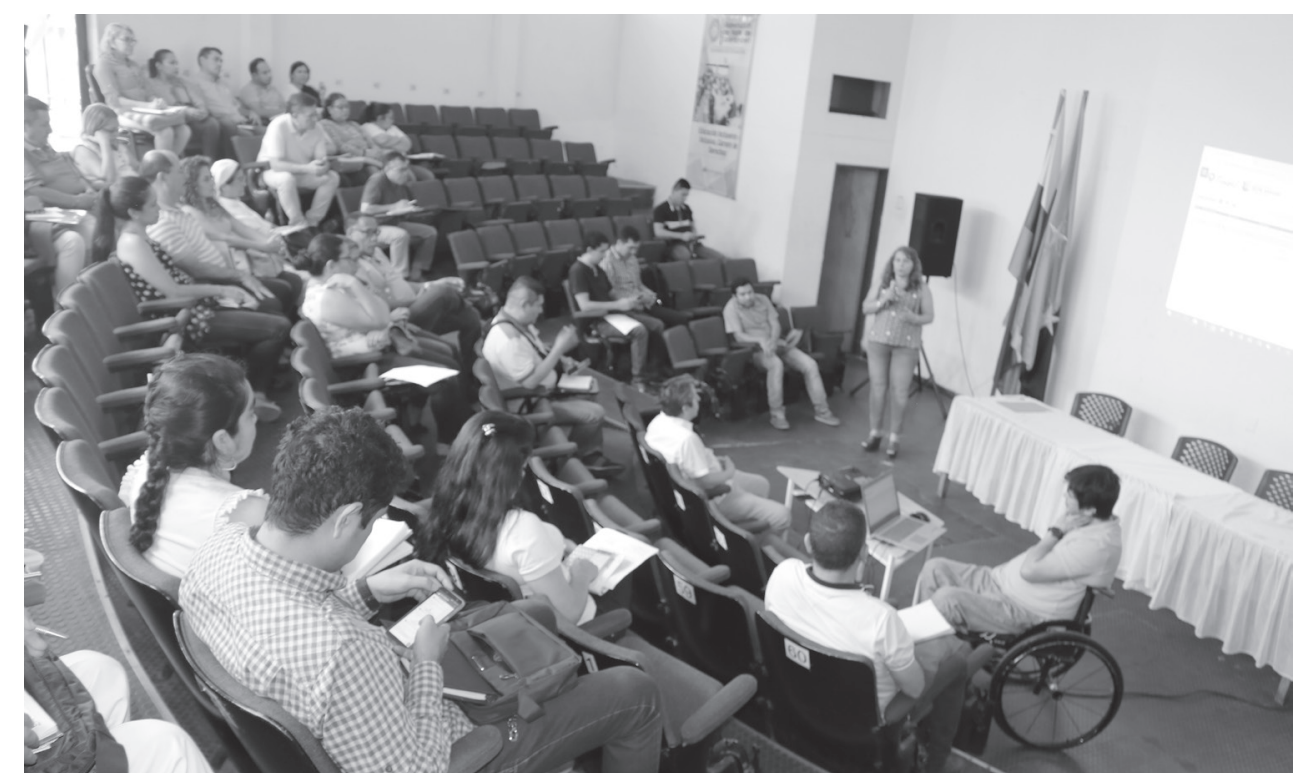

\section{Resumen}

El artículo presenta los resultados de un ejercicio de indagación de noticias alusivas al sector educativo publicadas en dos medios de comunicación de alta circulación en el norte de Colombia. El Heraldo es un periódico de amplia consulta entre líderes de opinión en ciudades como Barranquilla, mientras que la audiencia del Diario La Libertad se concentra más entre clases populares y municipios de la región.

Citar este artículo como: Parra, J. D. y Correa Hernández, G. (2018). Reflexiones sobre los retos de la política educativa en el Caribe colombiano: una indagación en prensa. Revista Papeles, 10(19), 56-70.

Fecha de recibido: 22 de febrero de 2018

Fecha de aceptación: 20 de mayo de 2018

* Doctor en Ciencias Sociales por el International Institute of Social Studies (ISS), Erasmus University Rotterdam. MA en Economía de Universidad de los Andes. Autor correspondiente. Correo electrónico: jparrah@ gmail.com

*\% Historiador de la Universidad del Atlántico. Correo electrónico: correa.perseo.gustavo@gmail.com 
El contraste de temáticas y debates difundidos en ambas fuentes da cuenta de las complejidades detrás del proceso educativo que surge tras hitos como la publicación de la Ley 715 de 2001. Los hallazgos del estudio sacan a relucir implicaciones metodológicas relevantes que invitan a la innovación en los debates académicos y políticos sobre los grandes retos educativos del país.

Palabras clave: política educativa, análisis de medios, calidad educativa, metodología de investigación.

\begin{abstract}
The article presents the results of a content analysis of news pieces about education published in two main periodicals from northern Colombia. El Heraldo is a newspaper that targets, mainly, opinion leaders from cities like Barranquilla, while the audience of the Diario La Libertad concentrates, widely, in popular classes and inhabitants from municipalities in the region. The exercise of contrasting themes and debates disseminated in both sources reveals the complexities behind education policy processes that emerged from events such as the expedition of Law 715 of 2001. The findings from this study highlight some methodological considerations that invite researchers and politicians to innovate in debates regarding the educational challenges of the country.
\end{abstract}

Keywords: education policy, media analysis, quality of education, research methodology.

\title{
Introducción: el objetivo del estudio
}

El problema de la denominada calidad de la educación básica y media en Colombia ha merecido una cantidad considerable de publicaciones académicas y de debates políticos en las últimas dos décadas (García-Villegas, Espinosa, Jimenez y Parra, 2013; Parra, 2015; Parra, 2018). A pesar de que ello puede generar la sensación de que el tema se encuentra sobre diagnosticado, persisten problemas metodológicos profundos en las prácticas de investigación dominante en la materia, y que permiten problematizar dicha premisa (Parra, 2018). Dicha situación puede verse reflejada en la reciente declaración de una académica colombiana:

[c]ada vez que el Ministerio de Educación ha querido mejorar la calidad de la educación, ha utilizado la misma vieja fórmula (...). Luego el Ministerio se pregunta por qué, después de años de esfuerzos, la calidad de la educación no mejora (Montoya-Vargas, 2016, p. 29).

El presente artículo representa un esfuerzo de innovación en el campo de la investigación de la educación y las políticas públicas. Este encuentra motivación en la búsqueda de rutas analíticas más promisorias a las de la tradición cuantiativa de insumo-producto dominante en dicho campo de estudio ${ }^{1}$, con el fin de avanzar

\footnotetext{
Pese a las repetidas aclaraciones que hacen expertos sobre el reduccionismo que implica el equiparar calidad educativa y desempeño escolar, la tradición cuantitativa que estudia el tema desde la academia tiende a reforzar dicha equivalencia. Dicho lo anterior, trabajos como el de Parra (2018) discuten la forma en que la tradición econométrica en el campo de estudio del desempeño (o la efectividad) escolar en Colombia (y a nivel internacional), hace uso de esquema insumo (ej. docentes, materiales)producto (ej. notas en exámenes estandarizados)
} 
en la acumulación de nuevo conocimiento que proponga debates en el sector. La propuesta se hace a partir de una indagación en prensa escrita, y se encuentra sustentada en la premisa de que el estudio de los discursos de los medios de comunicación puede proporcionar ideas importantes para comprender las dinámicas políticas, económicas y sociales de las reformas educativas contemporaneas (Waldowa, Takayamab y Sung, 2014). Lo anterior es visible en el acervo creciente de trabajos académicos en dicho frente (Thomas, 2002; Thomas, 2009; Stack, 2007; Green, 2015; Waldowa, Takayamab y Sung, 2014) y puede justificarse a partir de postulados metodológicos como el siguiente:

Recientemente, los académicos de la educación comparada han recurrido al análisis de los medios como una ruta para comprender las formas en que los contextos históricos, institucionales, culturales y políticos se combinan para influir en la política educativa. Por ejemplo, dado que la evaluación internacional se ha convertido en un tema de estudio en educación comparada, varios investigadores han recurrido al análisis de medios para ampliar su comprensión de cómo, por qué y bajo qué condiciones las evaluaciones internacionales se utilizan en diferentes contextos nacionales (Green, 2015, p. 29. Libre traducción de los autores).

Se discuten a continuación los resultados de un ejercicio de exploración de más de diez años de información publicada en dos medios de alta circulación en el Caribe colombiano: $E l$ Heraldo (EH) y el Diario la Libertad (LL). La lectura y también el contraste, del contenido de ambas fuentes de noticias, permiten estudiar

para identificar los determinantes del logro escolar. Esta representa una visión simplista del cambio educativo, el cual, según lo evidencia el análisis histórico, se caracteriza por procesos no lineales, no estandarizados, y demarcados por lógicas de conflictos de intereses entre actores sociales. elementos del discurso educativo al que tienen acceso dos segmentos claramento diferenciados de la población. Por ejemplo, mientras que $\mathrm{EH}$ es un medio ampliamente consultado por líderes (empresariales, de opinión) de la región caribe $^{2}$, el público objetivo de LL son "las clases populares y los municipios" (Coronel, 2016, p. 61). El periodo de consulta (20022014) representa, por tanto, una oportunidad para examinar diferentes perspectivas frente a la complejidad del proceso que surge tras la reforma educativa de la Ley 715 de 2001.

Dicha ruta metodológica responde a principios epistemológicos del campo de estudio de la economía política de la educación. Algunas investigaciones enmarcadas en dicha tradición, y que se han propuesto examinar transformaciones educativa en países europeos (Archer, 2013; Gonzáles, 2013) y en América Latina (Grindle, 2004), son enfáticas en la importancia de examinar a fondo las "interconexiones que se producen entre los diferentes actores sociales, para comprender cómo y por qué se han originado y modificado los sistemas educativos" (Gonzáles, 2013, p. 1215). Frente a dicha meta analítica habría que advertir que es claro que no es posible agotar el estudio de las interacciones entre seres humanos a partir de la lectura de información que estos (posiblemente) consultan (Parra, 2016; Parra, 2018) ${ }^{3}$.

2 Según los resultados de una encuesta que se publican en EH mismo, "el 33\% de los líderes de Atlántico consulta este medio de comunicación" Fuente especificada no válida. Conversaciones con representantes del sector educativo permiten corroborar el hecho que el EH es mucho más consultado, relativamente hablando, que LL por líderes empresariales y de gremios que mueven la inversión y la economía de ciudades como Barranquilla.

3 Archer (2013) señala que para poder estudiar por qué sucede $\mathrm{X}$ en el mundo (ej. por qué se presentan buenos indicadores educativos en una región) es importante analizar tanto elementos estructurales (ej. la disponibilidad de recursos materiales para invertir en el sector, las nociones culturales sobre el papel de la educación en una sociedad) y de la agencia de actores (ej. las motivaciones y las acciones 
En tal medida, este artículo hace apenas una contribución a dicho debate a partir de un esfuerzo por visualizar los marcos de referencia a partir de los cuales diferentes actores políticos del norte de Colombia construyen su visión, y sus preferencias, frente al cambio (o al estatus quo) educativo.

En términos de la estrategia del estudio, habría que advertir que existe un debate académico frente a la capacidad de los medios de comunicación de generar o de simplemente reflejar las percepciones de la ciudadanía, de los gobiernos y de líderes de opinión (Hopkins, Kim y Kim, 2017). Si bien la literatura especializada no es concluyente al respecto, existe cierto consenso sobre el poder que tiene la comunicación de hechos noticiosos sobre la forma en que se llegan a entender temas de interés público (Green, 2015). Esta última consideración es de particular interés para la presente reflexión, la cual busca hacer una aproximación a las preferencias o expectativas sobre el sector educativo de diferentes sectores de la población. En tal sentido, esta no se trata de una investigación sobre discursos educativos en medios escritos; su intención es la de caracterizar imaginarios colectivos que den pistas (al menos indicativas) sobre ejes temáticos relevantes para fundamentar nuevas agendas de investigación educativa.

\section{En términos de la estrategia del estudio, habría que advertir que existe un debate académico frente a la capacidad de los medios de comunicación de generar o de simplemente reflejar las percepciones de la ciudadanía, de los gobiernos y de líderes de opinión.}

\section{La organización del archivo de prensa y la selección de una muestra para el análisis}

La recolección de información en prensa hizo parte del trabajo de campo para una investigación doctoral sobre el estudio de las causas del bajo desempeño escolar en el Caribe colombiano. La consulta de medios fue hecha en el

efectivamente tomadas por directivos, docentes, estudiantes, etc.) y la forma en que interactúan en medio de, por ejemplo, la política educativa de un país. En este caso, se sugiere que la lectura de prensa refleja, hasta cierto punto, aspectos de la percepción (ej. la subjetividad) de diferentes actores del sector, y no necesariamente sobre cuestiones de contexto (de la estructura) donde surgen dichas percepciones o interpretaciones. Dicho lo anterior, para poder construir un argumento causal habría que contrastar los hallazgos de este documento con análisis más profundos sobre las estructuras educativasy el sistema educativo nacional.
Archivo Histórico de Barranquilla por dos historiados profesionales. A diferencia de otros medios escritos del país, ni EH ni LL cuentan con un registro electrónico organizado para la consulta en línea de sus ediciones relevantes para el periodo seleccionado (años 2002 a 2014), hecho que significó un trabajo manual de lectura de notas de prensa y almacenamiento de fotos de estas en un archivo digital.

Para efectos de la consulta, el equipo de investigación (integrado por los dos autores de este documento) construyó una lista de términos relevantes que fundamentaron una estrategia de búsqueda enfocada en la lectura de títulos y subtítulos sobre eventos educativos registrados como notas de prensa, excluyendo columnas de opinión. El listado incluía desde 
Gráfica 1. Frecuencia de notas de prensa encontradas en cada medio escrito consultado

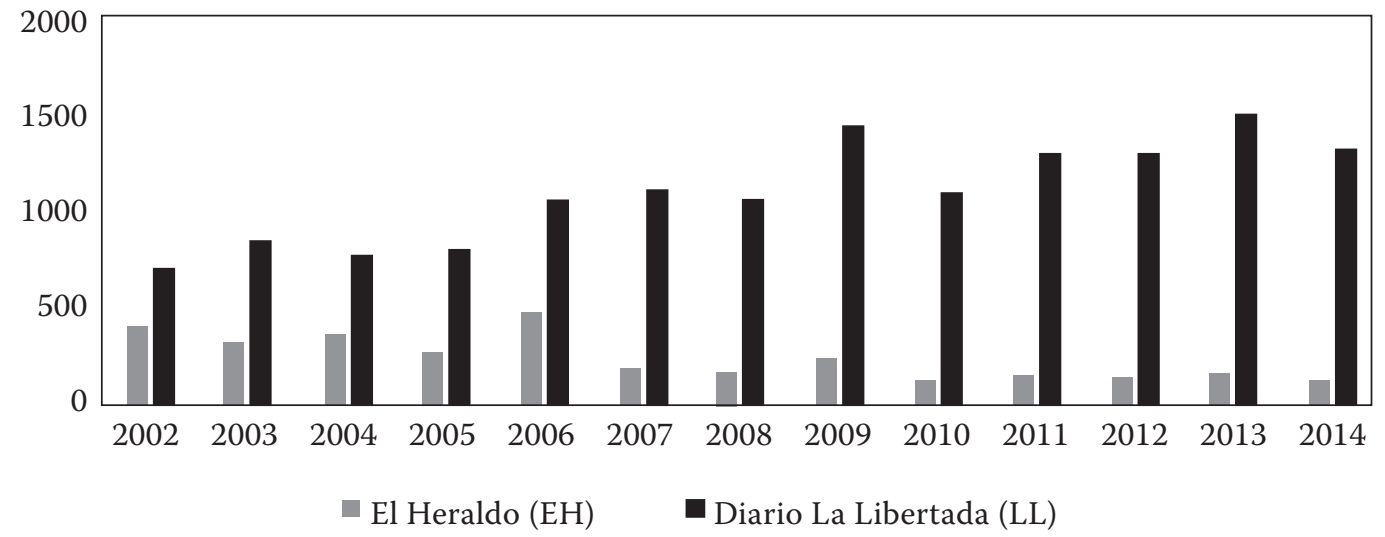

Fuente: elaboración propia

palabras genéricas como educación, institución educativa, política educativa, hasta nombres de instituciones - como el Ministerio de Educación o el ICFES- y programas concretos que proveen servicios educativos en el país - como Familias en Acción-. Para ampliar el criterio de búsqueda -y también la posibilidad de estudiar aspectos relevantes sobre dinámicas que hacen referencia a la educación básica y media- el listado incluyó términos alusivos a la educación superior (ej. Universidad, SENA $)^{4}$.

La gráfica 1 resume la frecuencia de eventos noticiosos recogidos $(\mathrm{N}=18.239)$. Dichos tabulados se hicieron a partir de una primera matriz de información consultada, donde se les pidió a los investigadores de archivo que, para cada nota de prensa fotografiada

4 La consulta se hizo solamente para ocho meses del año. Conversaciones con informantes clave motivaron tomar la decisión de excluir de la muestra de consulta los meses diciembre, enero, febrero y junio de cada año. Los tres primeros meses concuerdan, generalmente, con la época de carnaval en la región, hecho que afecta el flujo de actividades de instituciones educativas y de autoridades del sector. Junio, por su parte, es un mes de descanso de estudiantes y docentes, lo cual indica, nuevamente, una menor frecuencia de actividades (relativa a de los otros meses) que podrían generar hechos noticiosos. y almacenada, ingresaran el año, el mes y el día de la noticia, y datos como la página del periódico en que venía publicada y el total de páginas de cada edición. La organización del archivo permitió llevar a cabo una primera lectura descriptiva de la base de datos que indica que el volumen porcentual de información alusiva al campo educativo publicada en cada medio varía de manera sustancial. Según datos de la gráfica 2, LL registra en cada año más del doble (y en ocasiones más de cuatro veces) de eventos educativos que $\mathrm{EH}$, y dicha brecha se va acrecentando en el tiempo.

Para cada año de información se aplicaron pruebas estadísticas de diferencia de medias (pruebas $t$, pruebas Mann Whitney) que corroboraron que todas son estadísticamente significantivas.

Este primer hallazgo es interesante, en particular si se lee en paralelo con algunas investigaciones académicas. Por ejemplo, según Castelló-Climent (2008), la capacidad de una democracia de integrar las necesidades y los intereses de las mayorías en las decisiones políticas y económicas de un país depende, en gran medida, de qué tan distribuido se encuentre el capital humano (medido en de años educativos) en la sociedad. La lectura del análisis comparado de Soifer (2015) permite 
Gráfica 2. Porcentaje de noticias educativas en cada medio consultados (\%)

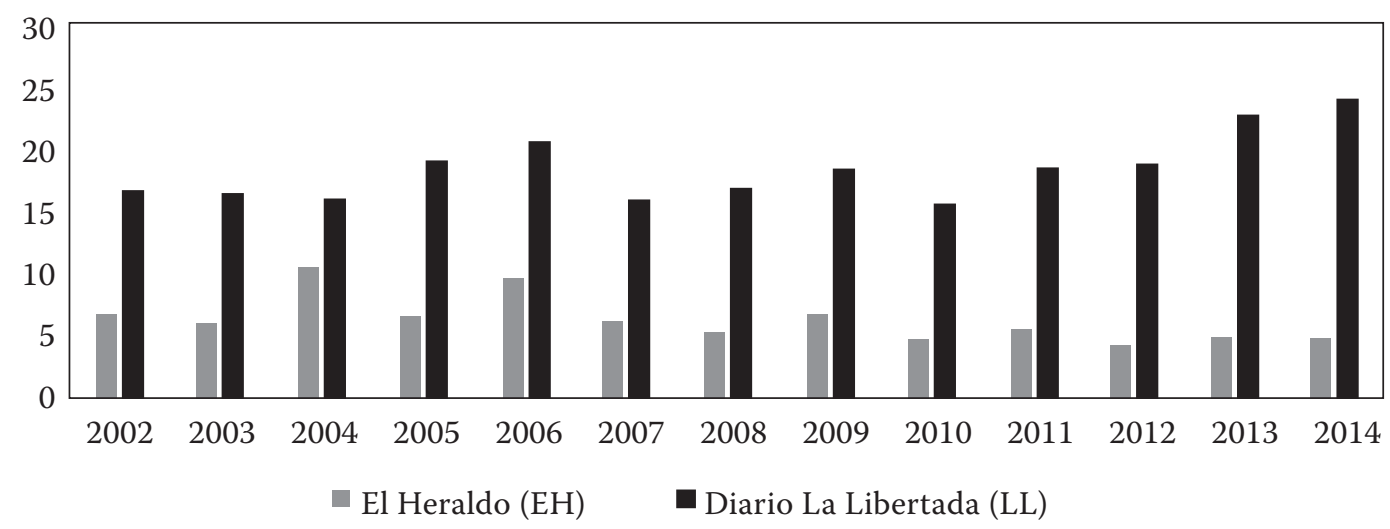

Fuente: elaboración propia.

discutir que, para el caso de Colombia, la falta de distribución de oportunidades educativas en el territorio se explica, en parte, por la falta de voluntad que han tenido las élites regionales para vincularse a un proyecto educativo nacional. Lo anterior permitiría plantear la hipótesis que los rezagos en materia de educativa que experimenta el Caribe colombiano se asocian al poco interés (relativo) que muestran sus empresarios y líderes de opinión en debatir sobre el tema.

Para la segunda etapa del análisis se seleccionó una submuestra para estudiar los contenidos de la información publicada. Para ello se tomó un mes de cada año consultado (el mismo para ambos periódicos) a partir de un cómputo para elegir meses que tuvieran una cantidad similar de noticias registradas en la base de datos. El promedio de noticias mensuales (sumando ambas fuentes) es de 175.4 y la desviación estándar es de 43.4. Al dividir este segundo dato en tres y sumarlo/ restarlo al primero se obtuvo un rango $(160.91 \leq \mathrm{x} \leq 189,8)$ que permitió delimitar meses elegibles en cada año. Para la selección final (ver anexo) se buscó, a su vez, escoger, dentro lo posible, una agrupación que permitiera contar con información relacionada con cada uno de los meses del año. Dicho ejercicio de muestreo arrojó un total de 2,256 noticias (559 pertenicientes EH y 1,590 LL) equivalentes a cerca del doce por ciento del universo de noticias.

El ejercicio analítico procedió con la aplicación de técnicas de análisis de contenido (Schreier, 2012; Green, 2015), con el apoyo del Software NVIVO. El formato de la información (fotografías) implicó un reto operativo adicional, en tanto dichos documentos no permiten hacer lecturas de tendencias a partir de análisis sobre uso de términos o frecuencias de palabras, como lo han propuesto otros académicos (Caicedo, Gaviria y Moreno, 2012). En esta etapa se hizo una lectura de cada noticia y se clasificó según variables como tipo de educación a la que hacía referencia (básica y media o superior, si hacía referencia a la región Caribe o a otras regiones, etc.). Junto a cada foto se escribió una descripción del contenido de la nota que sirvió, al final del recorrido por las más de dos mil notas de prensa, para clasificar los hechos noticiosos en categorías emergentes del contenido mismo de la información registrada. Se presentan a continuación algunos hallazgos de este ejercicio analítico, el cual buscó soporte en estudios sobre la política educativa en la región (Henríquez, 2013; Parra, 2017) para ayudar a los analistas a interpretar algunos patrones visualizados en los datos. 


\section{Debates en la prensa del Caribe colombiano: una aproximación al imaginario colectivo sobre las metas y objetivos de las políticas en educación}

Las tablas 1 y 2 resumen el ejercicio de análisis de información de más de diez años de prensa en dos medios de alta circulación en la Costa Caribe. Este permitió agrupar las noticias consultadas en 28 categorías que reflejan tendencias en temáticas discutidas y que a su vez permiten entrever posibles tensiones entre las percepciones y los intereses de diferentes actores educativos. En cada tabla la intensidad del color busca visualizar la importancia relativa (o la prioridad otorgada) entendida como su peso porcentual -que es el número en cada casilla- dentro del flujo total de información reportada anualmente. Ambas tablas hacen también una discriminación de datos por tipos de educación. En la medida que existen tópicos de política educativa en la que ambos niveles educativos se entrelazan de forma explícita (ej. la Universidad del Atlántico es una instancia asesora de la Secretaría de Educación Departamental, las universidades cuentan con programas especiales para la formación docente y de directivos de colegios oficiales) se hace relevante explorar el papel de las instituciones de educación superior en el sistema educativo regional.

En términos generales, en cuanto a educación básica y media, las categorías que más resaltan en $\mathrm{EH}$ son los anuncios de entregas de

\section{En la medida que existen tópicos} de política educativa en la que ambos niveles educativos se entrelazan de forma explícita, se hace relevante explorar el papel de las instituciones de educación superior en el sistema educativo regional. infraestructura y los problemas y necesidades no relacionadas con docentes en instituciones educativas (IE). En LL, por su parte, se hace un mayor énfasis en la discusión sobre políticas y programas educativos nacionales (tema también recurrente en EH), en el anuncio de acreditaciones de instituciones educativas y calificaciones de estudiantes (que incluye referencias a pruebas nacionales e internacionales para medir el desempeño escolar) y en el anuncio de seminarios y eventos académicos. En educación superior, si bien ambos medios dedican un espacio importante a la difusión de eventos académicos y de la oferta de programas de estudio, EH hace un énfasis en el reportaje de los problemas financieros de la educación pública y LL registra más (o al menos con mayor frecuencia a lo largo del tiempo) tópicos como protestas y manifestaciones públicas.

La discusión del resto de esta sección se centra en el desarrollo de una especie de proposiciones que surgen de la lectura de las tendencias en la información. En su conjunto, y como se discutirá con más detalle, estás temáticas aportan elementos para debatir la hipótesis propuesta por autores como Gamson y Modigliani (1989) y Chong y Druckman (2007) frente al papel dominante que tiene la opinión de élites políticas, sociales y económicas en la forma en que se presentan y, eventualmente, se abordan retos de la educación en el debate público. Es también interesante resaltar la forma en que la metodología del análisis permite estudiar rasgos de la historia reciente de la política educativa en Colombia y, tras ello, empezar a desmitificar narrativas dominantes según las cuales el proceso de la construcción de la agenda educativa nacional y regional ha sido un proceso lineal y fuertemente impulsado por aportes del debate tecnocrático (Parra, 2017). 


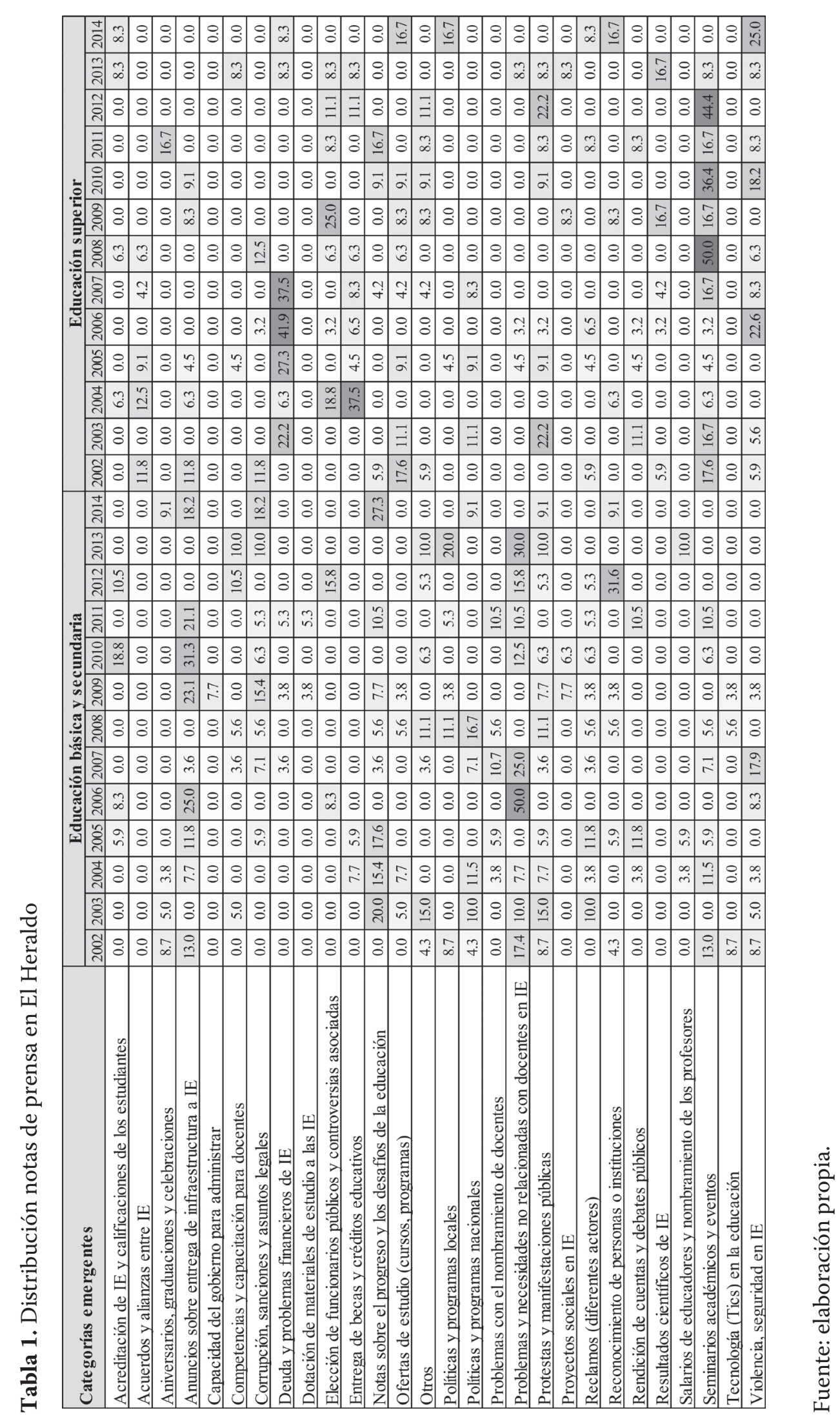




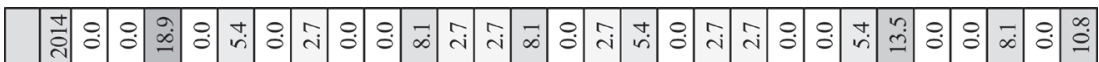

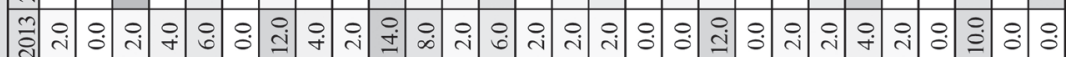

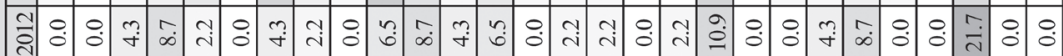

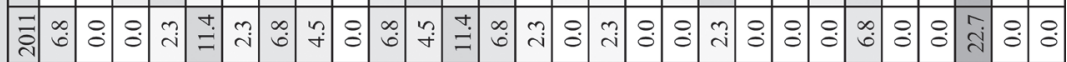

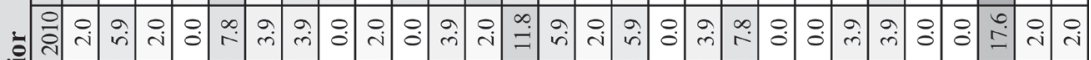

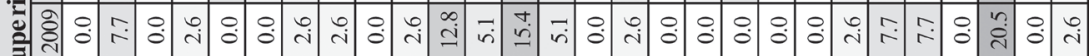

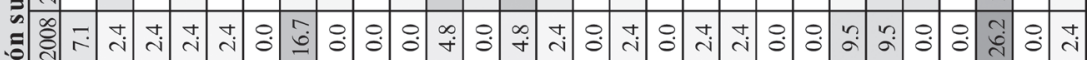

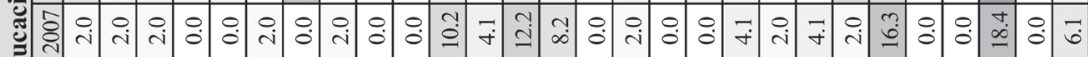

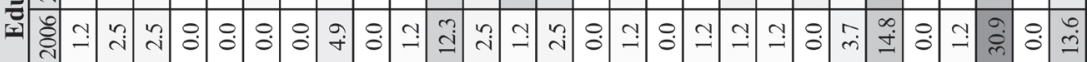

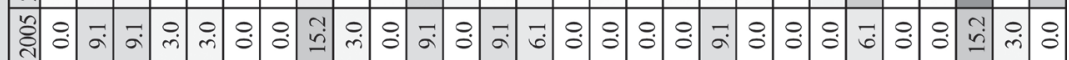

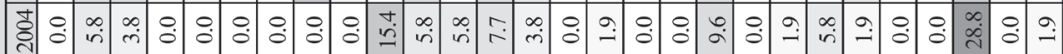

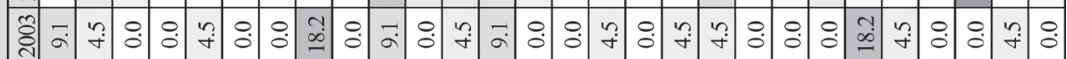
क्षे

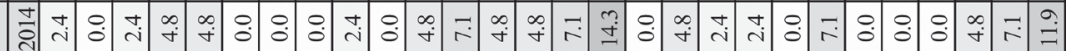

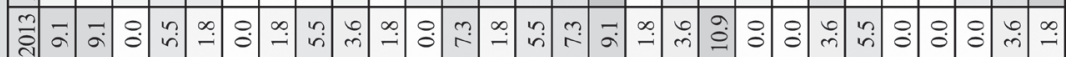

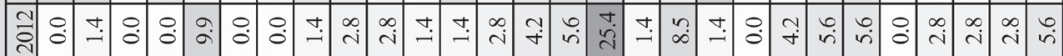

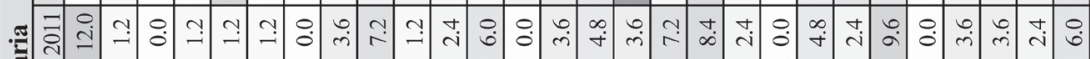

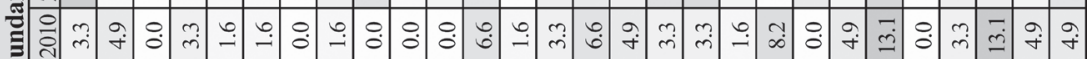

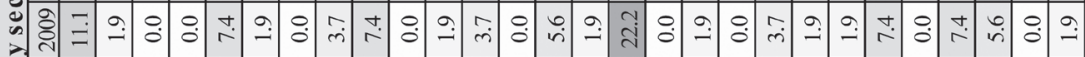

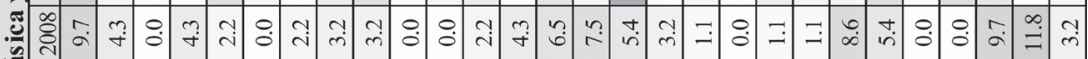

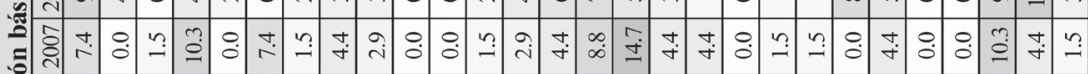

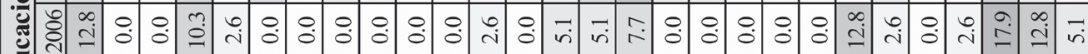

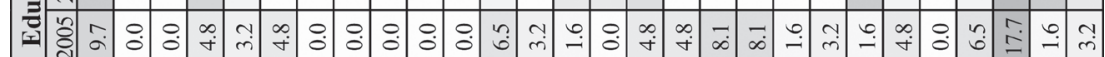

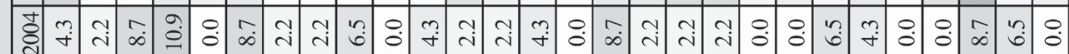
क्षी $\infty$ :

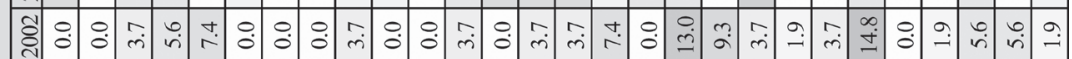

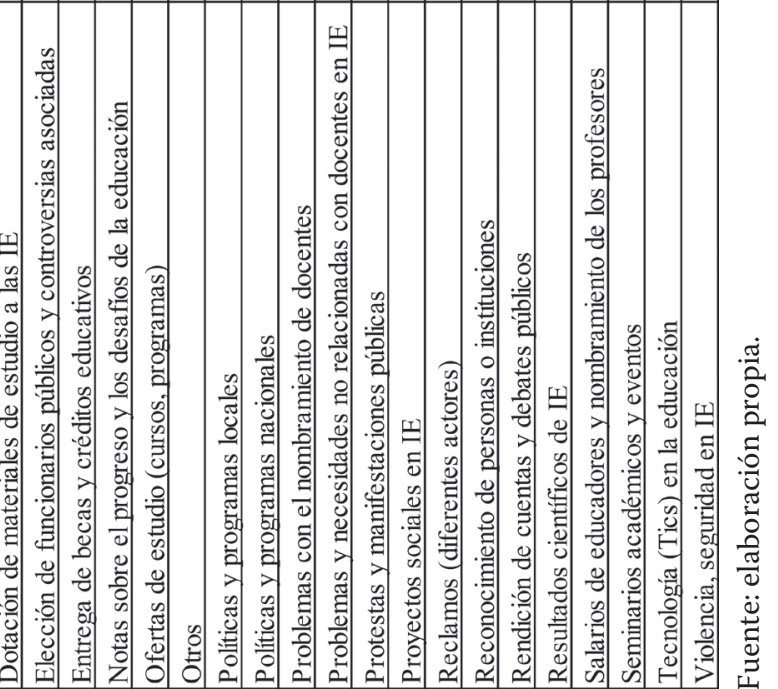




\section{La escasez de iniciativas educativas locales}

La cantidad de información que en ambos periódicos hace referencia a debates nacionales sobre política educativa duplica con creces las noticias sobre programas e iniciativas desde la región. En algunos casos, por ejemplo, los políticos visitan establecimientos educativos para entregar materiales y atender conmemoraciones - un caso común es el de la primera dama y otros representantes de ciudades como Barranquilla-. Este tipo de actos difícilmente son prueba de la efectividad en prácticas de planeación y ejecución de políticas locales. Paralelo a ello, una cantidad importante de las noticias reportadas hacen referencia a proyectos al interior de colegios (ej. la celebración del Día del Agua) que poco tienen que ver, cabe mencionarlo, con debates como la planeación curricular en contexto. Las escasas referencias a temas de ese tipo (ej. la necesidad de enfocarse en necesidades particulares de estudiantes en diferentes contextos) se dan cuando expertos hablan sobre generalidades de la pedagogía (ej. el uso de juguetes con niños) o una discusión en 2007 liderada por el MEN sobre proyectos pedagógicos en escuelas normales.

Existe otra categoría relevante para indagar sobre la construcción de diagnósticos regionales: notas sobre el progreso y los desafíos de la educación. Esta incluye, por ejemplo, editoriales, pero también notas en otras secciones de los periódicos, que refuerzan una y otra vez la idea de que un gran problema de la educación en el Caribe es que no satisface estándares nacionales. Es ilustrativo el caso,

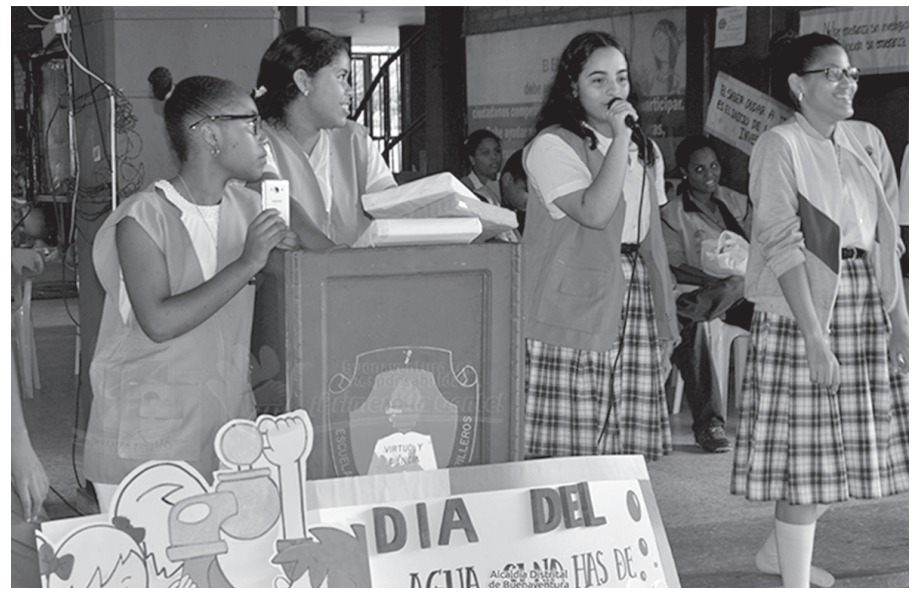

por poner uno, de una pieza noticiosa de LL donde la máxima autoridad del ICFES en la región declara que una gran prioridad en su nuevo cargo es el de alinear su agenda con directrices del MEN. Buscar alineación con lógicas nacionales no es algo negativo per se; el punto es que, al leer dicha tendencia junto a declaraciones de diferentes actores regionales (Parra, 2017), esta cobra un sentido de pasividad frente a la priorización de problemas específicos del norte del país. Esta última percepción encuentra resonancia en otra noticia del mismo periódico, pero esta vez del año 2014, donde un educador galardonado con un premio de excelencia docente (el Premio Compartir) reflexiona sobre cómo evaluaciones como las pruebas PISA - que tanto inquietan a líderes de opinión - fallan en capturar las complejidades educativas a lo largo del territorio nacional.

Existe otra categoría relevante para indagar sobre la construcción de diagnósticos regionales: notas sobre el progreso y los desafíos de la educación. Esta incluye, por ejemplo, editoriales, pero también notas en otras secciones de los periódicos, que refuerzan una y otra vez la idea de que un gran problema de la educación en el Caribe es que no satisface estándares nacionales. 


\section{Las necesidades de las instituciones educativas}

El debate sobre la llamada excelencia docente es uno que ha cobrado gran protagonismo en la agenda educativa nacional e internacional de las últimas dos décadas (Coffield, 2012; Parra, 2015). Si bien el resultado de este ejercicio no permite invalidar la hipótesis sobre el papel fundamental de la formación y las capacidades de los educadores en el aprendizaje de los estudiantes, sí desafía la lógica que la inversión en este elemento sería suficiente para resolver muchos de los retos de los colegios oficiales de la región. La categoría de problemas y necesidades no relacionadas con docentes en las IE (ej. los problemas básicos de infraestructura, de acceso a servicios públicos) es la más representada en EH en los años 2002, 2006, 2007, 2012 y 2013, con picos hasta del $50 \%$. En LL el tema también es visible, pero con menos frecuencia.

Es interesante anotar cómo la categoría competencias y capacitación para docentes tiene una mención relativamente baja en ambos medios, con excepciones en años más recientes de información de EH y de los años 2004 y 2007 de LL. La discusión sobre el tema en educación superior se da, pero también con una baja intensidad. Al adentrase en los contenidos de las noticias, existen reportajes sobre diferentes actividades de formación a educadores, incluyendo, por ejemplo, intercambios académicos (en su mayoría) en Estados Unidos y en particular para el refuerzo de la segunda lengua. Ambos medios resaltan (al menos de forma implícita) la importancia del tema de la excelencia docente, pero difícilmente reportan algún debate sobre la racionalidad de (o la lógica que deberían tener) los programas de formación docente sobre necesidades concretas de aprendizaje de estudiantes. $\mathrm{Al}$ respecto, valdría la pena resaltar una noticia que apareció en 2014 en LL que menciona un estudio de la Universidad Nacional de Colombia sobre la forma en que las discusiones de política educativa en el país siempre privilegian asuntos financieros y administrativos sobre aspectos pedagógicos y curriculares. En el mismo año, EH publicó una nota señalando cómo los malos resultados en la Pruebas PISA se conectan, de forma directa, con la calidad docente.

\section{Promesas (in)cumplidas de la modernización en capacidades de gestión del sector}

De acuerdo con miradas sobre la historia reciente de la educación en la región Caribe ${ }^{5}$, es factible identificar una transición, en algún punto hacia la mitad de la década del año dos mil, en el énfasis de su política educativa. Este migra de un esfuerzo por fortalecer capacidades instaladas de autoridades educativas regionales (ej. la Secretaría de Educación del

Como parte del trabajo de campo del proyecto, se organizó un grupo focal con supervisores de educación del Departamento del Atlántico. Dicha actividad se propuso a dibujar una línea de tiempo de la historia reciente de la política educativa en la región, entre 2002 y 2014. Ver Parra (2017).
Atlántico) a un enfoque sobre la planeación educativa a partir del reconocimiento de los problemas al interior de instituciones educativas. La categoría de capacidad del gobierno para administrar agrupa notas alusivas al tópico y, como tal, su baja aparición en la información analizada podría aportar elementos para problematizar la narrativa de la modernización de las capacidades administrativas en el sector - debate en el cual existen otros aportes académicos (Henríquez, 2013; Parra, 2017).

En este respecto, es relevante mencionar cómo EH tiene una cobertura casi inexistente del tema, con una excepción de casi ocho por 
ciento de las noticias en 2009. En LL la tendencia muestra picos de noticas reportadas en el 2002, el 2009 y el 2012 y muestra que, en efecto, tiende a haber una transición de temas reportados como la búsqueda de certificación de la Secretaría de Educación, como la de Soledad y la reorganización de plantas de personal y de capacidades para monitorear a instituciones educativas, a discusiones sobre el fortalecimiento de capacidades instaladas y del liderazgo (ej. por parte de rectores) en instituciones oficiales. Ello contrasta, sin embargo, con el contenido de categorías como la de los problemas de los colegios no relacionados con la capacidad docente (mencionado arriba) o la cantidad no despreciable de notas alusivas a protestas y manifestaciones, y que sugeriría la persistencia de necesidades no resueltas en el sector. En términos del contenido de las noticias, cabe resaltar una nota en EH en 2007 donde el MEN denuncia la alta rotación de funcionarios públicos en secretarías de educación, y una de 2012 en LL donde se denuncia la baja capacidad de autoridades locales para evitar que algunas instituciones incumplan las leyes de gratuidad ${ }^{6}$.

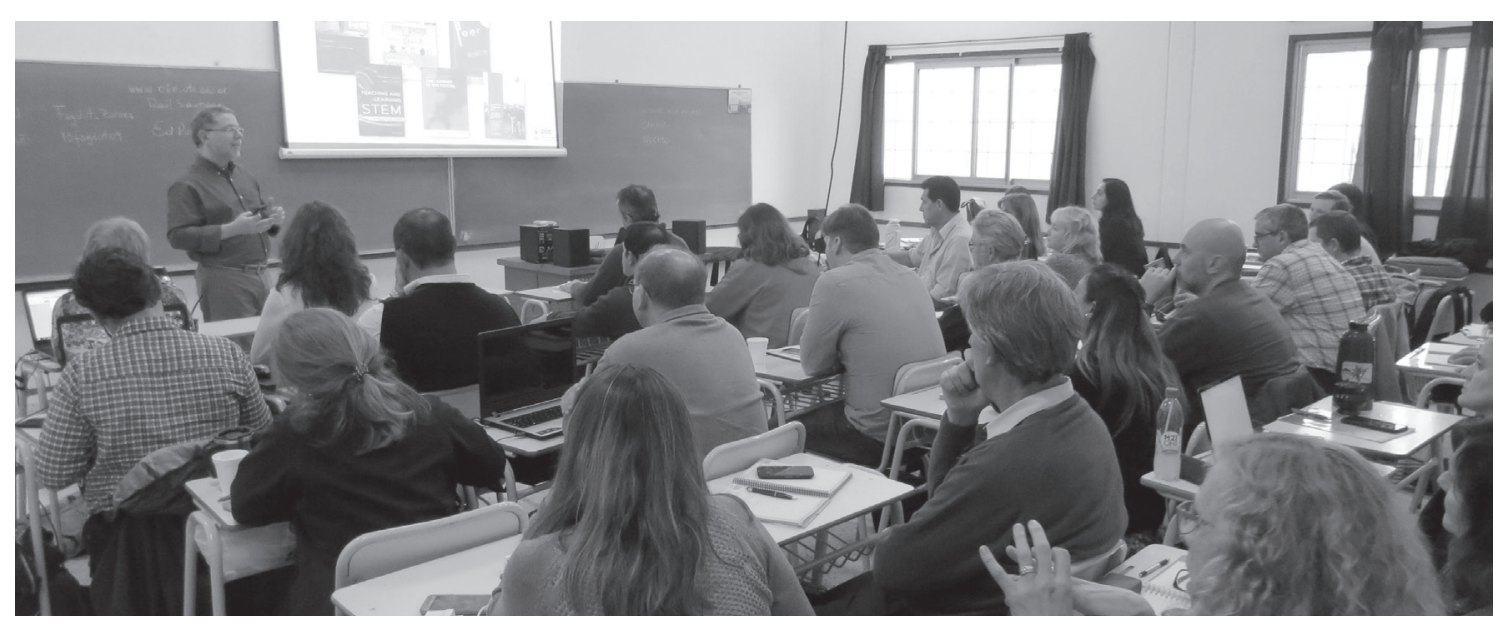

\section{Los colegios: ¿una amalgama homogénea o una cuestión de diversidad?}

Finalmente, es relevante resaltar la diferencia en la forma en que ambos medios consultados tipifican (o enmarcan) la institución educativa. Las tendencias en cuanto a tipos de información reportada en cada medio son indicativas al respecto. Como se señaló ya al inicio de esta sección, mientras que EH dedicó en el periodo estudiado la mayoría de su espacio (relativo) para reseñar asuntos como la entrega de servicios e infraestructura y los problemas no docentes en colegios, LL resalta (más) otros temas como los eventos, la acreditación de instituciones, los debates de política educativa tanto local como nacional y la implementación de tecnologías de la información en aulas de clase. El contenido mismo de las noticias indica que este segundo medio no solo resalta problemáticas sociales (la categoría de problemas no asociados a docentes sigue siendo relevante), pero también cuenta con un espacio importante - al menos mayor que su contraparte- para informar sobre aspectos como nombramiento de funcionarios y directivos docentes, sobre reconocimientos a personas (ej. premios a docentes) y sobre

6 Según el artículo dos del Decreto 4807 de 2011, "La gratuidad educativa se entiende como la exención del pago de derechos académicos y servicios complementarios. En consecuencia, las instituciones educativas estatales no podrán realizar ningún cobro por derechos académicos o servicios complementarios" 
La invitación a académicos y expertos es a poner más atención al debate sobre la economía política en el sector, sin el cual es difícil entender por qué algunas medidas técnicas (ej. la formación docente) parecen no arrojar los resultados esperados.

temáticas concretas que afectan a actores educativos particulares, como es el caso de la violencia contra los educadores.

Una interpretación que se puede dar a dicho patrón de datos es que LL exalta más que EH la diversidad en el sector educativo. En el caso del segundo medio se podría hablar de cierta tendencia a presentar los colegios como instituciones ciertamente homogéneas y cuyo debate puede agotarse hablando sobre problemas genéricos (ej. infraestructura, falta de útiles) y soluciones ciertamente estandarizadas (ej. entrega de servicios). Al relacionar este

\section{Conclusión}

El contraste entre visiones expresadas en dos medios de comunicación con audiencias claramente diferenciadas permite resaltar diferencias importantes en la forma en que distintos segmentos poblacionales conciben y expresan sus preferencias frente al sector educativo. En términos generales, $\mathrm{EH}$, que es el medio de los líderes empresariales y de opinión, tiende a crear una narrativa sobre la educación básica y media fundamentada en la visión de la escuela como una entidad homogénea, con necesidades comunes (ej. los problemas de infraestructura) y con problemas generalizados frente a la calidad de la enseñanza. LL, que es un medio consultado por el público más amplio, exalta más la diversidad al interior de las instituciones educativas de la región, dedicando espacio a documentar historias sobre docentes, directivos y otros actores educativos locales.

Dicho ejercicio, a pesar de ser exhaustivo en términos de la cantidad de información hecho con la información sobre su audiencia objetivo (ej. en su mayoría empresarios, líderes de opinión), podría alimentarse la hipótesis que para $\mathrm{EH}$ existen menos incentivos para adentrarse en las complejidades del día a día del sector educación. El caso de LL es distinto. este periódico no solo dedica más espacio (relativo) a reportar sobre los retos de la educación básica y media (y también superior), sino que, a pesar de evidenciar también situaciones de necesidad en las instituciones que prestan servicios educativos, se toma más tiempo para reflexionar sobre las dinámicas del sector y de sus protagonistas. analizada, no permite, por sí solo, hacer conclusiones en torno a interrogantes causales como, por ejemplo, los determinantes de la persistencia del bajo desempeño escolar en el norte del país. Sin embargo, las tendencias analizadas admiten plantear hipótesis referentes a las características del proceso político detrás de la definición de estrategias y líneas de acción para buscar cambios educativos en la región. Por ejemplo, siguiendo el planteamiento del párrafo anterior, es posible que los malos resultados en indicadores educativos de un departamento como el Atlántico (Parra, 2017) se expliquen por los pocos incentivos con los que cuentan élites empresariales y líderes de opinión para fomentar debates (ej. sobre el currículo escolar, sobre la pedagogía en aulas de clase) e inversiones educativas que respondan a necesidades específicas de niños y niñas del Caribe colombiano.

En términos metodológicos, el texto representa un esfuerzo por innovar en el debate sobre la 
investigación en política pública educativa nacional. El mensaje no es el de desconocer los aportes de investigadores en campos como la economía de la educación, frente a los factores causales (ej. la excelencia docente) que son determinantes para fomentar cambios educativos en Colombia. La invitación a académicos y expertos es a poner más atención al debate sobre la economía política en el sector, sin el cual es difícil entender por qué algunas medidas técnicas (ej. la formación docente) parecen no arrojar los resultados esperados.

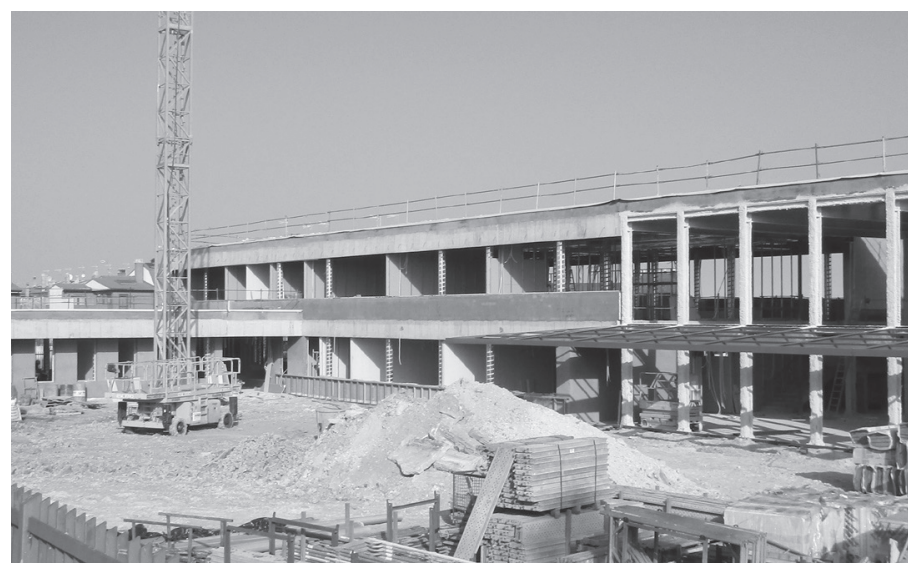

\section{Referencias}

Archer, M. (2013). Social Origins of Educational Systems. Londres y Nueva York: Routledge.

Caicedo, J., Gaviria, A. y Moreno, J. (2012). Hechos y palabras: la realidad colombiana vista a través de la prensa escrita. Revista de Economía Institucional, 26, 137-168.

Castelló-Climent, A. (2008). On the distribution of education and democracy. Journal of Development Economics, 87, 179-190.

Chong, D., \& Druckman, J. (2007). A Theory of Framing and Opinion Formation in Competitive Elite Environments. Journal of Communication, 57, 99-118.

Coffield, F. (2012). Why the McKinsey reports will not improve school systems. Journal of Education Policy, 27(1), 131-149.

Coronel , H. (2016). Observatorio de medios del caribe colombiano. Santa Marta: Universidad Sergio Arboleda.

El Heraldo. (03 de 11 de 2017). El Heraldo, el diario más influyente en su región. Barranquilla, Colombia. Obtenido de https://www. elheraldo.co/barranquilla/el-heraldo-el-diariomas-influyente-en-su-region-418850

Gamson, W., \& Modigliani, A. (1989). Media Discourse and Public Opinion on Nuclear Power: A Constructionist Approach. American Journal of Sociology, 95(1), 1-37.
García-Villegas, M., Espinosa, R., Jimenez, F. y Parra, J. (2013). Separados y desiguales: Educación y clases sociales en Colombia. Bogotá: Editorial Dejusticia.

Gonzáles, M. (2013). Teoría social realista y la construcción del sistema educativo estatal en España: algunas propuestas para el análisis del cambio educativo. Revista Mexicana de Investigación Educativa, 18(59), 1213- 1239.

Green, N. (2015). Analyzing Public Discourse: Using Media Content Analysis to Understand the Policy Process. Current Issues in Comparative Education, 18(1), 26-41.

Grindle, M. (2004). Despite the odds. The contentious politics of Education Reform. Princeton y Oxford: Princeton University Press.

Henríquez, H. (2013). Politicas públicas en educación y desarrollo en la Región Caribe. Revista de Economía del Caribe, 12, 217-246.

Hopkins, D., Kim, E. y Kim, S. (2017). Does newspaper coverage influence or reflect public perceptions of the economy? Research and politics, 4(4), 1-7.

Montoya-Vargas, J. (2016). El Campo de los estudios curriculares en Colombia. Bogotá: Universidad de los Andes.

Parra, J. D. (2015). The Paradigm of Critical Realism and Involving Educators in Policy 
Debates. GIST Education and Learning Jour$\operatorname{nal}(10), 149-171$.

Parra, J. D. (2016). Realismo crítico: una alternativa en el análisis social. Sociedad y Economía(31), 215-238.

Parra, J. D. (2017). El reto de la descentralización educativa: reflexiones desde la mirada de actores nacionales y subnacionales en el caso del departamento del Atlántico. Papel político, 22(2), 339-367.

Parra, J. D. (2018). Critical realism and school effectiveness research in Colombia: The difference it should make. The British Journal of Sociology of Education, 39(1), 107-125.

Schreier, M. (2012). Qualitative Content Analysis . Londres: Sage.

Soifer, H. (2015). State Building in Latin America. Nueva York: Cambridge University Press.

Stack, M. (2007). Representing School Success and Failure: media coverage of international tests. Policy Futures in Education, 5(1), 100-110.

Thomas, S. (2002). Contesting education policy in the public sphere: media debates over policies for the Queensland school curriculum. Journal of Education Policy, 17(2), 187-198.

Thomas, S. (2009). Newspapers on education policy: constructing shared public discourses on curriculum, schools and teachers. In W. Housley, \& R. Fitzgerald, Media, Policy and Interaction (pp. 205-223). Londres: Ashgate Publishing.

Waldowa, F., Takayamab, K., \& Sung, Y. (2014). Rethinking the pattern of external policy referencing: media discourses over the "Asian Tigers" PISA success in Australia, Germany and South Kore. Comparative Education, 50(3), 302-321.
Anexo. Muestra de noticias para análisis

\begin{tabular}{|l|l|c|}
\hline Año & \multicolumn{1}{|c|}{ Mes } & Noticias \\
\hline 2002 & Abril & 162 \\
\hline 2003 & Abril & 172 \\
\hline 2004 & Mayo & 180 \\
\hline 2005 & Septiembre & 169 \\
\hline 2006 & Octubre & 163 \\
\hline 2007 & Agosto & 178 \\
\hline 2008 & Noviembre & 180 \\
\hline 2009 & Julio & 161 \\
\hline 2010 & Septiembre & 182 \\
\hline 2011 & Marzo & 189 \\
\hline 2012 & Octubre & 189 \\
\hline 2013 & Marzo & 170 \\
\hline 2014 & Abril & 161 \\
\hline Total & & $\mathbf{2 , 2 5 6}$ \\
\hline
\end{tabular}

Fuente: elaboración propia

\section{Sobre financiamiento}

El presente artículo hace parte de una investigación doctoral sobre la persistencia del bajo desempeño escolar en regiones de Colombia. El proyecto es financiado por el programa de becas para doctorados en el exterior de Colciencias y un apoyo para estudios doctorales de la firma Econometría Consultores.

\section{Nota de agradecimiento}

Los autores agradecen a Steven Bolgueman y a Juan Carlos Llerena por su excelente labor como asistentes de investigación. Las conversaciones con Eugénie Richard, investigadora de la Universidad Externado de Colombia, fueron fundamentales para la definición de la ruta metodológica del estudio. Un agradecimiento especial a ella. 